\title{
WASP, Tregs, and food allergies - rare disease provides insight into a common problem
}

\author{
Yun Liang and Johann E. Gudjonsson \\ Department of Dermatology, University of Michigan, Ann Arbor, Michigan, USA.
}

\begin{abstract}
Dysregulation of the type 2 immune system presents with various manifestations, including allergic inflammation, and has emerged as an alarming public health issue. The pathological mechanisms that underlie Thelper type 2 cell-driven (Th2-driven) allergic diseases remain unclear. In particular, it is not completely understood how type 2 immunity is restricted in inflammatory responses. In this issue of the $\mathrm{JCl}$, Lexmond et al. use Wiskott-Aldrich syndrome as a model disease and establish that the Wiskott-Aldrich gene product (WASP) serves an essential role in $T$ regulatory cells to contain Th2 effector cell differentiation and prevent allergic sensitization to dietary antigens.
\end{abstract}

Immune imbalance in WiskottAldrich syndrome

The Wiskott-Aldrich syndrome (WAS) is a rare, but severe, $\mathrm{X}$-linked recessive disease characterized by the triad of thrombocytopenia, eczema, and immune deficiency that frequently leads to pyogenic and viral infections. WAS is caused by mutations in the WAS gene. A milder form of WAS known as X-linked thrombocytopenia (XLT) is also associated with WAS gene mutations but is mainly characterized by a bleeding tendency. The WAS gene product (WASP) is a cytoplasmic protein that serves as an integrator of cell surface signaling cascades, such as those downstream of the $\mathrm{T}$ cell antigen receptor (TCR), and the actin cytoskeleton network. WASP is mainly expressed in hematopoietic cell lineages (1).

Given that WASP is involved downstream of the TCR, it is interesting that Th1 and Th2 development is differentially sensitive to WAS loss (2). While $\mathrm{CD} 4^{+}$and $\mathrm{CD} 8^{+}$ $\mathrm{T}$ cells from WAS patients display reduced secretion of the Th1 cytokines IFN- $\gamma$ and TNF- $\alpha$, these patient $\mathrm{T}$ cell populations exhibit a minimal disruption of the production and secretion of Th2 cytokines, such as IL-4 (3). This imbalance between Th1 and Th2 responses in WAS patients likely relates to the development of eczema that is characteristic of this syndrome. In this issue, Lexmond et al. show that patients with mutations in the WAS gene (both WAS and XLT) frequently develop IgE-mediated reactions to common food allergens and demonstrate using Was knockout mice that loss of WASP in T regulatory cells (Tregs) leads to unrestrained Th2 effector responses that drive allergic intestinal inflammation (Figure 1) (4).

\section{Relationship of immunodeficiency syndromes to Th2 and IgE responses}

Dysregulated Th2 responses, atopy, and elevated $\operatorname{IgE}$ levels are observed in a variety of human immunodeficiency diseases, including IPEX (immune dysregulation, polyendocrinopathy, enteropathy, X-linked, which is caused by functional defects in FOXP3), hyper-IgE syndrome, Omenn syndrome, and atypical complete DiGeorge syndrome. The pathological mechanisms and consequences of elevated IgE in these conditions are poorly understood but likely involve several distinct immunological pathways, including increased Th2 cyto-

Related Article: p. 4030

Conflict of interest: J.E. Gudjonsson has received research support from Amgen, AnaptysBio, and Novartis

Reference information: J Clin Invest. 2016;126(10):3728-3730. doi:10.1172/JCI90198.

kine production, particularly in WAS, IPEX, and Omenn syndrome (5).

Aside from Th2 cells, other inflammatory cells, including innate lymphoid cells, eosinophils, basophils, and mast cells, contribute to type 2 immune responses. Key mediators involved in type 2 reactions include the cytokines IL-4 and IL-13. Type 2 immune responses have been shown to protect against multicellular parasites, suppress sustained Th1-driven inflammation, promote tissue repair, and regulate metabolic responses. Dysregulation of the Th2 response has been linked to a wide range of diseases, particularly allergic disorders, uncontrolled infections, tumorigenesis, and fibrosis (6). Th2 cytokines and costimulatory signals promote B cell class-switch recombination and production of antigen-specific IgE. Binding of antigen-specific IgE antibodies to FceRI on mast cells leads to degranulation. Under IgE stimulation, mast cells also contribute to the late-phase reaction by secreting mediators that recruit leukocytes and sustain Th2-associated inflammation, resulting in tissue remodeling (7). This relationship accounts for the critical role that has been shown for IgE and mast cells in allergic disorders.

\section{Food antigen sensitization in WAS and role of Tregs}

Lexmond and colleagues evaluated 25 patients with WAS mutations and revealed an increased sensitization and prevalence of food allergy among these individuals compared with the general population. Moreover, $\mathrm{Was}^{-/}$mice exhibited spontaneous sensitization to food antigens accompanied by increased levels of IgE along with intestinal mast cell expansion and degranulation. In a WASP-deficient mouse model, the sensitization was independent of the commensal microbial community, although the isotype composition of the humoral anti-food response had some influence. Interestingly, targeted WASP deficiency specifically targeted to $\mathrm{FOXP3}^{+}$Tregs resulted in height- 
A

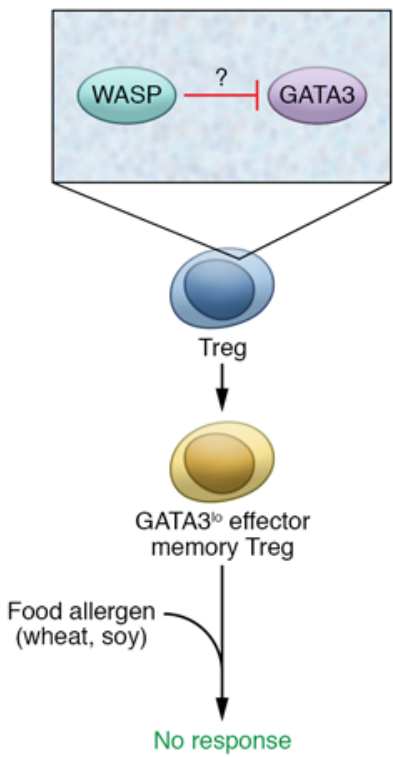

B

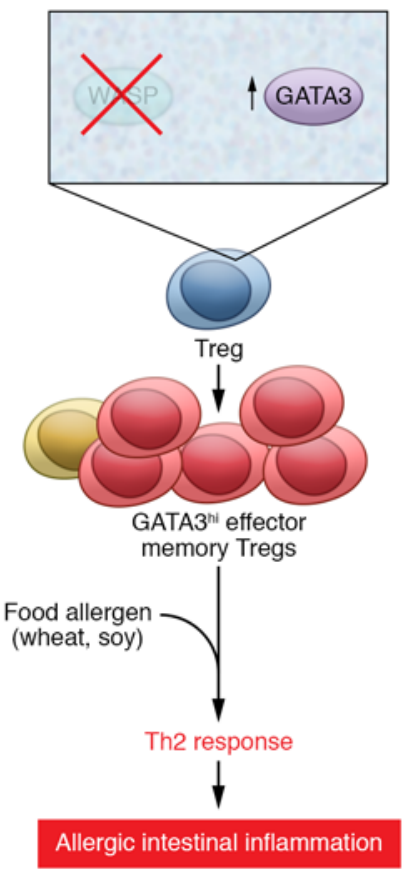

Figure 1. WASP functions in Tregs to contain Th2-mediated allergy. (A) In WASP-expressing Tregs, GATA3 expression is repressed and thereby restrains Th2-type inflammation in response to food antigens. (B) Tregs lacking WASP, such as occurs in patients with mutations that lead to Wiskott-Aldrich syndrome and $\mathrm{X}$-linked thrombocytopenia, are insufficient in restraining Th2-type inflammation in response to food allergens. This lack of Th2 suppression is associated with an increased frequency of CATA $3^{\text {hi }}$ effector memory Tregs.

ened food allergy responses and more severe disease, as measured by small intestinal inflammation and increased expression of Th2 cytokines. In contrast, conditional deletion of Was in $\mathrm{B}$ cells or $\mathrm{CD}_{11 \mathrm{c}^{+}}$ dendritic cells had no effect. These results establish a major role of Tregs in regulating Th2 responses, tolerance to food allergens, and mast cell expansion in the gut (4).

\section{WASP regulates Treg function}

Several lines of evidence obtained from WASP-deficient mice have pointed to a role for WASP in regulation of proliferation and tissue homing of Tregs (8-11). However, there has not been a direct investigation into the consequences of the conditional deletion of Was in Tregs. While lack of WASP did not appear to influence the absolute numbers of FOXP3 $^{+}$Tregs, WASP-deficient Tregs assumed a Th2-like phenotype, as determined by elevated expression of the transcription factor GATA3, which promotes Th2 differentiation, but suppresses Th1 differentiation, in naive T cells. Tregs with a GATA3 ${ }^{+}$, Th2-like phenotype have been implicated in oral allergic sensitization and promotion of food allergies in both mice and humans (12). Consistent with a role for these cells in allergic responses, numbers of GATA $3^{+}$Tregs were increased in patients with WAS gene mutations (4). Interestingly, the pathogenicity of individual WAS mutations positively correlated with the level of GATA $3^{+}$Tregs in the blood, further supporting the relevance of this Treg subset in allergic inflammation and its association with WASP function.

\section{Remaining questions and future directions}

This connection between the increase in GATA $^{+}$Tregs and unrestrained Th2 response is exciting; however, several questions remain to be answered. What are the functional characteristics of these GATA3 ${ }^{+}$ Tregs - are they defective in their ability to contain Th2 responses and/or do they directly promote Th2-type inflammation? What are the mechanisms underlying the functional differences between GATA $^{\mathrm{h}}{ }^{\mathrm{h}}$ versus GATA3 ${ }^{\text {lo }}$ Tregs? Moreover, what insights could be gained from studying the transcriptional targets of GATA3 in Tregs? It has previously been suggested that nearly $70 \%$ of GATA 3 targets in Tregs and Th2 cells overlap (13), raising the possibility that GATA $^{+}$Tregs may have a profile and function similar to that of Th2 cells. What, then, are some important distinctions in the GATA3-regulated transcriptional profiles of the two cell types? Does FOXP3, the Treg master transcriptional regulator of Tregs, play a role the delineation of these cells, given that FOXP3 acts together with GATA3 on some target genes in Tregs (14)? Future studies to address these questions will be exciting to follow and see what the answers to these questions will be.

In summary, the work by Lexmond et al. demonstrates that WASP in FOXP3 ${ }^{+}$ Tregs plays a role in restraining Th2-mediated food allergy, and shows that a cell-specific process in a rare disease may be translatable to more common conditions. Apart from food allergies, further elucidation of the molecular mechanism by which WASP regulates Treg function, and how the aberrant function of Tregs leads to allergic sensitization may yield important insights into other allergic diseases, such as asthma. It will be exciting to see whether the same processes play a role in allergic sensitization in other organs, for example, the skin in conditions such as atopic dermatitis (15). The study by Lexmond and colleagues provides exciting insights into the biology of Th2-driven diseases and may facilitate development of therapeutic approaches.

\section{Acknowledgments}

Johann E. Gudjonsson is supported by NIH grant R01-AR069071, and the A. Alfred Taubman Medical Research Institute Frances and Kenneth Eisenberg Emerging Scholar Award and Doris Duke Charitable Foundation grant 2013106. Yun Liang is supported by NIH grant T32-AR007197.

Address correspondence to: Johann E. Gudjonsson, 1910 Taubman Center, 1500 E. Medical Center Drive, Ann Arbor, Michigan 48109, USA. Phone: 734.615.4508; E-mail: johanng@med.umich.edu.

\footnotetext{
1. Thrasher AJ, Burns SO. WASP: a key immunological multitasker. Nat Rev Immunol. 2010;10(3):182-192.

2. Matalon O, Reicher B, Barda-Saad M. WiskottAldrich syndrome protein--dynamic regulation of actin homeostasis: from activation through
} 
function and signal termination in Tlymphocytes. Immunol Rev. 2013;256(1):10-29.

3. Trifari S, et al. Defective Th1 cytokine gene transcription in $\mathrm{CD}^{+}$and $\mathrm{CD} 8^{+} \mathrm{T}$ cells from Wiskott-Aldrich syndrome patients. J Immunol. 2006;177(10):7451-7461.

4. Lexmond WS, et al. FOXP3 ${ }^{+}$Tregs require WASP to restrain Th2-mediated food allergy. JClin Invest. 2016;126(10):4030-4044.

5. Ozcan E, Notarangelo LD, Geha RS. Primary immune deficiencies with aberrant IgE production. J Allergy Clin Immunol. 2008;122(6):1054-1062.

6. Wynn TA. Type 2 cytokines: mechanisms and therapeutic strategies. Nat Rev Immunol. 2015;15(5):271-282.
7. Galli SJ, Tsai M. IgE and mast cells in allergic disease. Nat Med. 2012;18(5):693-704.

8. Maillard MH, et al. The Wiskott-Aldrich syndrome protein is required for the function of CD4(+)CD25(+)Foxp3(+) regulatory T cells. JExp Med. 2007;204(2):381-391.

9. Humblet-Baron S, et al. Wiskott-Aldrich syndrome protein is required for regulatory $\mathrm{T}$ cell homeostasis. J Clin Invest. 2007;117(2):407-418.

10. Marangoni F, et al. WASP regulates suppressor activity of human and murine $\mathrm{CD} 4(+) \mathrm{CD} 25(+)$ FOXP3(+) natural regulatory T cells. J Exp Med. 2007;204(2):369-380.

11. Adriani M, et al. Impaired in vitro regulatory $\mathrm{T}$ cell function associated with Wiskott-Aldrich syndrome. Clin Immunol. 2007;124(1):41-48.
12. Noval Rivas M, et al. Regulatory T cell reprogramming toward a Th2-cell-like lineage impairs oral tolerance and promotes food allergy. Immunity. 2015;42(3):512-523.

13. Wohlfert EA, et al. GATA3 controls Foxp3(+) regulatory $\mathrm{T}$ cell fate during inflammation in mice. J Clin Invest. 2011;121(11):4503-4515.

14. Rudra D, et al. Transcription factor Foxp3 and its protein partners form a complex regulatory network. Nat Immunol. 2012;13(10):1010-1019.

15. Spergel JM, Mizoguchi E, Brewer JP, Martin TR, Bhan AK, Geha RS. Epicutaneous sensitization with protein antigen induces localized allergic dermatitis and hyperresponsiveness to methacholine after single exposure to aerosolized antigen in mice. JClin Invest. 1998;101(8):1614-1622. 